\title{
GRUPOS DE REFLEXÃO E AÇÃO: UMA INTERVENÇÃO PSICOTERAPÊUTICA FEMINISTA COM MULHERES VÍTIMAS DE VIOLÊNCIA NA INTIMIDADE
}

\author{
Sofia Neves ${ }^{1}$, Carla Cunha ${ }^{2}$,Helena Grangeia ${ }^{3}$, Ariana Correia ${ }^{4}$
}

Instituto Universitário da Maia

\section{Resumo}

Descreve-se neste texto a implementação e avaliação de um programa de intervenção psicoterapêutica de cariz feminista junto de quatro grupos de mulheres vítimas de violência na intimidade. Tendo como objetivos gerais 1) informar sobre o fenómeno da violência de género, 2) desconstruir discursos de género, 3) refletir em torno dos significados e dos impactos das histórias pessoais de vitimação e 4) promover o empowerment e a capacitação pessoal e social, o programa foi desenvolvido com 23 mulheres com idades compreendidas entre os 29 e os 61 anos $(M=45.93$; $S D=9.13)$, provenientes de diferentes regiões da zona norte de Portugal.

A fim de se avaliar a eficácia do programa foram administrados o Inventário sobre Vivências Violentas nas Relações Íntimas, a Escala de Progresso Pessoal Revista e o The Outcome Questionnaire-45.2. Os resultados sugerem progressos muito positivos quanto ao bem-estar psicológico e ao empowerment das participantes.

Palavras-chave: empowerment; violência de género; terapia de grupo; vítimas de violência na intimidade.

\begin{abstract}
Reflection and Action Groups: A therapeutic program with women victims of intimate violence

This paper describes the implementation and assessment of a feminist psycotherapeutic intervention with four groups of women victims of partner violence. The main goals guiding this intervention were: 1 ) to inform about the phenomenon of gender violence, 2) to deconstruct discourses on gender, 3) to reflect on meanings and the impact of personal trajectories of victimization and 4) promote empowerment and personal and social agency. This program was conducted with four groups of women, coming from different regions in the North of Portugal, in a total of 23 participants aged between 29 and 61 years old $(M=45.93 ; S D=9.13)$. For assessing the effectiveness of this group intervention, several measures were applied: the Inventory of Violent Experiences in Personal Relationships, the Personal Progress Scale and the Outcome Questionnaire-45.2. Results evidence significant gains in the psychological well being and empowerment of these participants.
\end{abstract} lence.

Keywords: empowerment; gender violence; group therapy; victims of partner vio-

\author{
Instituto Universitário da Maia (ISMAI). asneves@docentes.ismai.pt \\ Instituto Universitário da Maia (ISMAI). ccunha@docentes.ismai.pt \\ Instituto Universitário da Maia (ISMAI). hgrangeia@docentes.ismai.pt \\ Instituto Universitário da Maia (ISMAI). ariana.pinto.correia@gmail.com
}




\begin{abstract}
Resumé
Groupes de réflexion et d'action: une intervention thérapeutique avec femmes victimes de violence conjugale

Cet article décrit la mise en œuvre et l'évaluation d'un programme de psychothérapie féministe avec quatre groupes de femmes victimes de violence dans les relations intimes. Les objectifs du programme sont: 1) l'information sur le phénomène de la violence de genre, 2) la déconstruction des discours de genre, 3) la réflexion sur les significations et les impacts des histoires personnelles de victimisation et 4) la promotion du empowerment et de la capacitation personnelle et sociale des femmes. Le programme a été mis en œuvre avec 23 femmes âgées entre 29 et 61 ans $(M=45.93 ; S D=9.13)$. Afin d'évaluer l'efficacité du même étaient administrés l'Inventaire des Expériences Violentes dans les Relations Intimes, l'Échelle de Progression Personnelle et le Outcome Questionnaire-45.2. Les résultats suggèrent des progrès très positif en ce qui concerne le bien-être psychologique et l'empowerment des participants.
\end{abstract}

Mots-clés: empowerment; violence entre les sexes; thérapie de groupe; victimes de violence dans les relations intimes.

\title{
A Violência contra as Mulheres na Intimidade
}

A violência contra as mulheres na intimidade é um flagelo à escala global. De acordo com a Agência dos Direitos Fundamentais da União Europeia (FRA, 2014), uma em cada três mulheres é vítima de violência pelo parceiro após ter completado os 15 anos de idade.

Fortemente determinada pela assimetria de poderes entre os sexos (Amâncio, 1994; Dias e Guerreiro, 2010; Neves, 2008) e por desigualdades estruturais de ordem variada (Bourdieu, 1999; Ferreira, 1998), a violência de género compreende um conjunto de processos e de dinâmicas que tem na sua base o sexismo e o patriarcado como formas de organização social. Não sendo a pertença de género despiciente na determinação dos comportamentos de vítimas e de agressores, a hierarquia de poder entre os sexos, configurada umas vezes tácita, outras explicitamente, legitima níveis diferenciados de dominação, particularmente no contexto da família e na esfera da intimidade.

Abrangendo «todos os atos que resultem, ou possam resultar, em danos ou sofrimentos físicos, sexuais, psicológicos ou económicos para as mulheres, incluindo a ameaça de tais atos, a coação ou a privação arbitrária da liberdade, tanto na vida pública como na vida privada» (Conselho de Europa, 2013: 4-5), a violência de género constitui uma grave violação dos direitos humanos e um sério problema de saúde pública (Lisboa et al., 2006).

Muito embora em Portugal a violência doméstica se tenha tornado um crime público em 2000, nos últimos 14 anos os avanços legislativos não se têm feito acompanhar de uma mudança efetiva de mentalidades. Refira-se a título ilustrativo que em 2013 foram registadas 27318 ocorrências (Ministério da Administração Interna, 2014), no âmbito das quais $81 \%$ das vítimas eram mulheres. No 
corrente ano e só no 1. semestre foram já assassinadas pelos seus companheiros ou ex-companheiros 24 mulheres (OMA, 2014).

Ainda que os números referidos anteriormente espelhem uma realidade dramática, eles são porventura uma ponta do icebergue da violência cometida contra as mulheres em Portugal. Muita desta violência não é reportada às autoridades, em muitos casos porque as vítimas não confiam na eficiência do sistema judicial, preferindo assim o silêncio à denúncia. Outras, aquelas que tomam iniciativa de denunciar, confrontam-se não raras vezes com as fragilidades dos instrumentos jurídicos normativos e com a descredibilização e/ou culpabilização dos profissionais que os aplicam. Estas e outras evidências sugerem que a violência de género, pela sua magnitude e complexidade, exige respostas prementes de todos os intervenientes sociais, num quadro global de políticas públicas centradas nos direitos das vítimas (Davies, Francis e Jupp, 2003). Tal preocupação vai ao encontro do espírito da Convenção de Istambul e do V Plano Nacional de Prevenção e Combate à Violência Doméstica e de Género (2014-2017), que impõem uma abordagem integrada e multidisciplinar na prevenção e combate ao fenómeno.

No campo da Psicologia tais respostas podem dar-se em várias frentes (Walker, 1999). A ação psicoterapêutica tem demonstrado ser um recurso promotor da garantia dos direitos das vítimas, na medida em que as consciencializa para a necessidade de acionarem mecanismos internos e externos de validação das suas experiências. No sentido de educar para os direitos humanos e de promover o bem-estar das mulheres vítimas de violência na intimidade, foram desenvolvidos e implementados os GRA, cujo racional se descreve em seguida.

\section{Os Grupos de Reflexão e Ação (GRA)}

Os GRA são uma proposta de intervenção psicoterapêutica em grupo com mulheres vítimas de violência na intimidade de cariz feminista (Cortés, 2011; Enns, 2004; Loureiro, Pinheiro e Neves, 2012; Neves e Nogueira, 2003, 2004, 2005; Walker, 2000; Worell e Remer, 2003), desenvolvida em consonância com os pressupostos gerais dos Grupos de Ajuda Mútua (CIG, 2011). Esta modalidade de intervenção foi originalmente formulada no âmbito do Projeto n. ${ }^{\circ}$ 087961/2012/77 da Tipologia 7.7. do POPH, financiado pela Comissão para a Cidadania e Igualdade de Género (CIG).

A intervenção feminista, não sendo ideologicamente neutra e tendo como foco de análise as relações sociais de género, assume que a estrutura cultural e política condiciona a realidade das mulheres, propondo por isso a sua desnaturalização e desocultação (Cortés, 2011; Neves e Nogueira, 2003, 2004a, 2004b, 2005). Nesta ótica, os GRA baseiam-se numa perspetiva crítica e reflexiva que convida as vítimas a reposicionar-se face aos seus lugares de pertença social, colocando a ênfase na reflexão e ação através da promoção do empowerment. 
O empowerment, constructo introduzido nas Ciências Sociais por Rappaport em 1981, é um conceito desenvolvimental que visa descrever o potencial de crescimento e de autonomização dos indivíduos, das famílias e das comunidades (Rapport, 1984). Tradicionalmente associado às mulheres e a outros grupos socialmente marginalizados (Jones e Meleis, 1993), o empowerment tem sido definido como um processo de autodeterminação (Worell e Remer, 2003). Ao fomentar o empowerment através da aquisição de conhecimentos e de competências que promovam o coping efetivo com situações de desigualdade, discriminação, exclusão e stresse interpessoal e social, os GRA procuram, em última instância, a redução da sintomatologia clínica resultante dos efeitos da vitimação.

Os grupos, por seu turno, são espaços privilegiados de suporte social e de mudança, onde as aprendizagens são potenciadas pela identificação, pela partilha e pelo apoio mútuo. No caso das vítimas de violência na intimidade, os grupos parecem funcionar como contextos de validação emocional e de preparação para a mudança. Assim, os GRA perfilham quatro objetivos centrais: 1) informar sobre o fenómeno da violência de género, 2) desconstruir discursos de género, 3) refletir em torno dos significados e dos impactos das histórias pessoais de vitimação e 4) promover o empowerment e a capacitação pessoal e social.

\section{Método}

No presente estudo, pretendeu-se atingir os seguintes objetivos específicos: a) caracterizar o tipo e frequência da violência sofrida antes e depois dos GRA; b) avaliar a evolução da sintomatologia ao longo do tempo; c) avaliar a evolução do empowerment ao longo do tempo; d) explorar a relação entre o empowerment e a mudança sintomatológica ao longo do tempo.

\section{Participantes}

Os GRA foram implementados junto de quatro grupos constituídos, no total, por 23 participantes (seis no Grupo 1, cinco no Grupo 2, sete no Grupo 3 e cinco no Grupo 4), utentes de instituições de apoio a vítimas de violência na intimidade da zona norte do país .

A participante mais jovem tinha 30 anos e a menos jovem 61 anos $(\mathrm{M}=46.5$; $\mathrm{DP}=9.13$ ). Treze tinham habilitações literárias inferiores ao $9 . .^{\circ}$ ano, nove entre o $9 .$. e o 12. ${ }^{\circ}$ ano e uma tinha concluído uma licenciatura. Nove delas estavam desempregadas, uma reformada e as restantes treze empregadas. Uma era solteira, treze casadas, três estavam em união de facto e seis eram divorciadas. Apenas duas das participantes eram de nacionalidade estrangeira (uma brasileira e uma francesa). Todas as participantes tinham pelo menos um/a filho/a ( $\mathrm{M=1.57)}$, com idades compreendidas entre os 3 anos e os 39 anos. Três das 23 participantes resi- 
diam em contexto de casa abrigo, oito coabitavam com os agressores e doze residiam sozinhas ou com outros membros da família.

De acordo com a informação fornecida pelas próprias nas fichas sócio-biográficas, doze das 23 participantes eram, aquando da inclusão nos grupos, vítimas de violência pelos parceiros ou ex-parceiros. As participantes foram vítimas de violência pelos companheiros ou ex-companheiros, em média, 12 anos $(\mathrm{DP}=11.95)$, tendo sido a duração mínima da vitimação de dois meses e a duração máxima de 40 anos.

As vítimas foram alvo, em média, de dois tipos diferenciados de violência, sendo que 19 foram expostas a violência física (82.60\%), 23 a violência psicológica (100\%), 6 a violência sexual (26.08\%), 8 a violência económica (34.78\%) e 10 a violência social $(43.47 \%)$. Em 20 dos 23 casos foi apresentada denúncia contra o agressor, aguardando-se sentença em três casos. 18 das 23 participantes indicaram estar em acompanhamento psicológico individual aquando da sua integração nos grupos.

Registaram-se, no decurso do processo, cinco dropouts (dois no grupo 2 e um por cada um dos restantes grupos) e uma morte (no grupo 1). Esta foi provocada pelo ex-companheiro da vítima, seu alegado agressor, que se encontra atualmente detido, acusado de homicídio. O crime foi praticado na semana em que o grupo iria ver dinamizada a última sessão, o que fez com que a intervenção neste caso tivesse no total 11 sessões e não dez. Relativamente aos dropouts, estes deveram-se a motivos de saúde, autonomização e conflitos interpessoais entre participantes.

\section{Instrumentos}

Por forma a avaliar-se a eficácia do programa de intervenção foram administrados, nas sessões 0, 8 e de follow up (um mês após a finalização da intervenção), os seguintes instrumentos:

Inventário sobre Vivências Violentas nas Relações Íntimas (IVVRI; Versão para investigação de Neves, Cunha, Grangeia e Correia, 2014a).

Construído de raiz, o IVVRI é composto por 20 itens e mede a frequência de comportamentos de violência física, psicológica e sexual sofrida. Para cada um dos itens do IVVRI é solicitada uma de três respostas possíveis: nunca, só uma vez, mais do que uma vez (e em caso afirmativo se aconteceu no último ano). Na primeira fase de elaboração do instrumento, o mesmo foi sujeito a um processo de reflexão falada (Almeida e Freire, 1997).

Escala de Progresso Pessoal Revista (EPPR; Johnson, Worell e Chandler, 2005. Versão para investigação de Neves, Cunha, Grangeia e Correia, 2014b).

Concebida originalmente em 1998, por Worell e Chandler, esta escala tem sido usada para avaliar a eficácia da intervenção feminista, medindo o empower- 
ment. A escala avalia também os dez resultados genéricos da terapia feminista: 1) a autoavaliação, 2) o equilíbrio entre o conforto e o distresse, 3) a consciência dos papéis culturais e de género, 4) o autocontrolo e a eficácia pessoal, 5) o autocuidado, 6) as competências de resolução de problemas, 7) a assertividade, 8) o acesso aos recursos, 9) a flexibilidade cultural e de género e 10) o ativismo social.

Os 28 itens que constituem a EPPR são cotados numa escala de Likert de três pontos de «Nunca» a «Sempre».

The Outcome Questionnaire-45 (OQ-45.2; Lambert et al., 1996; Adaptado e traduzido por Machado e Fassnacht, no prelo).

O OQ-45.2 é um questionário de autorrelato que procura avaliar as mudanças dos/as clientes ao longo do processo psicoterapêutico. É composto por 45 itens, cotados em escalas tipo Likert de cinco pontos («Nunca» a «Quase sempre»), e permite avaliar o índice de sintomatologia geral e o progresso dos/as clientes. Integra quatro subescalas, designadamente 1) comportamentos de risco, 2) sintomatologia clínica, 3) relações interpessoais e 4) papéis sociais. Este instrumento, cuja pontuação global varia entre os 0 e os 180 pontos, tem revelado uma boa consistência interna em estudos prévios (e.g., $\alpha=.92$ no estudo de Ferreira e Matos, 2013). A versão portuguesa demonstrou boas qualidades psicométricas (Machado e Fassnacht, no prelo): usando a pontuação global, considera-se uma pontuação de 62 como ponto de corte para a indicação de sintomatologia clinicamente significativa e uma descida de 15 pontos na pontuação total como uma mudança fidedigna, de acordo com os critérios do reliable change index de Jacobson e Truax (1991).

Foi ainda preenchida na sessão 0, por cada uma das participantes, o consentimento informado e a ficha sócio-biográfica. A ficha sócio-biográfica procede a uma caracterização genérica dos dados sociodemográficos e do historial de vitimação passado e atual, complementando assim os dados do IVVRI.

\section{Procedimentos}

Depois de, numa primeira fase, se terem contactado as direções das instituições de apoio a vítimas, via telefone e/ou $e$-mail, explicando-se genericamente os objetivos do programa de intervenção e as condições da sua implementação, foram realizadas reuniões presenciais com todas elas no sentido da clarificação de eventuais questões. Acordou-se que a triagem inicial das potenciais participantes seria feita pela equipa técnica das respetivas instituições, de acordo com os critérios de inclusão previamente definidos, a saber: existência de historial de vitimação na intimidade, idade superior a 18 anos, ausência de indicadores de psicopatologia grave ou quadros psiquiátricos, ausência de quadros de dependência de substâncias, ausência de défices cognitivos severos e capacidade de compreensão e expressão da língua portuguesa. 
No total foram contactadas 55 potenciais participantes, tendo-se conseguido uma adesão inicial de 29 vítimas. Organizados em cinco módulos, os GRA foram compostos por oito sessões, com a duração média de $1 \mathrm{~h} 30 \mathrm{~m}$ cada. Para além destas foi ainda realizada a sessão 0 e a sessão de follow up. As sessões foram dinamizadas por uma terapeuta e por uma coterapeuta e decorreram nas instalações das instituições. A participação nos GRA não implicou nenhum tipo de encargo financeiro nem para as instituições, nem para as utentes.

Para a análise da estatística descritiva, comparação entre os grupos de participantes e exploração da relação entre as variáveis utilizou-se o programa SPSS (Statistical Package for Social Sciences - IBM Corp., 2013).

\section{Resultados}

\section{Caracterização dos comportamentos de violência sofrida pelas participantes}

Relativamente aos resultados do IVVRI, e analisando item a item (cf. tabela 1) nos três momentos da avaliação, verificou-se uma diminuição da prevalência de todos os comportamentos de violência sofrida do pré para o pós-teste, o que indicia uma menor exposição à vitimação aquando do término do processo de intervenção. Saliente-se a cessação de comportamentos de violência sexual indesejados ou forçados com recurso a ameaças, força física ou armas.

Observou-se igualmente uma diminuição da prevalência da violência do pós-teste para o follow up, nomeadamente no que respeita ao impedimento do contacto com a família e outros, à limitação e controlo dos gastos, às ameaças de morte, aos atentados contra a vida e à necessidade de receber tratamento hospitalar, ao controlo e à perseguição em espaços públicos, às ameaças verbais, aos atos de ignorar, desprezar, humilhar, envergonhar, tratar com indiferença, magoar fisicamente a vítima ou terceiros, impedir o sono, o descanso, a alimentação ou a higiene, estrangular, asfixiar, atropelar, ferir com gravidade e às ameaças com recurso a armas ou à força física. Um mês após a última sessão foi detetado um agravamento da violência emocional (culpar, criticar, insultar, difamar e acusar), das ameaças com recurso às tecnologias de informação e comunicação e do controlo (impedimento de trabalhar, estudar ou sair, controlo da forma de vestir, frequência de locais).

Verificou-se uma manutenção da cessação de comportamentos de violência sexual indesejados ou forçados com recurso a ameaças, força física ou armas. 


\section{Tabela 1}

Prevalência dos comportamentos de violência sofrida

\begin{tabular}{lccc}
\hline & Pré-teste & Pós-teste & Follow \\
& (Sessão 0) & (Sessão 8) & up \\
\hline Culpar/Criticar/Insultar/Difamar/Acusar & $96.6 \%$ & $34.5 \%$ & $37.9 \%$ \\
Ameaçar verbalmente & $93.1 \%$ & $27.6 \%$ & $24.1 \%$ \\
Magoar fisicamente & $89.6 \%$ & $13.8 \%$ & $13.7 \%$ \\
Ignorar/Desprezar/Humilhar/Envergonhar/Tratar com indiferença & $86.2 \%$ & $31 \%$ & $20.7 \%$ \\
Ameaças de morte/Atentado à vida/Necessidade de tratamento médico & $86.2 \%$ & $24,1 \%$ & $17.2 \%$ \\
Ameaçar com armas ou força física & $82.7 \%$ & $13.7 \%$ & $10.3 \%$ \\
Impedir sono, descanso, alimentação ou higiene & $72.4 \%$ & $20.7 \%$ & $17.2 \%$ \\
Controlar forma de vestir, frequência de locais & $72.4 \%$ & $17.2 \%$ & $27.5 \%$ \\
Mexer em pertences sem autorização & $68.9 \%$ & $17.2 \%$ & $17.2 \%$ \\
Comportamentos sexuais indesejados & $62.1 \%$ & $0 \%$ & $0 \%$ \\
Controlar/Perseguir em espaços públicos & $62 \%$ & $31 \%$ & $20.7 \%$ \\
Impedimento do contacto com família e outros & $62 \%$ & $13.7 \%$ & $6.9 \%$ \\
Magoar fisicamente terceiros & $58.6 \%$ & $13.8 \%$ & $13.7 \%$ \\
Limitação/Controlo dos gastos & $55.2 \%$ & $20.6 \%$ & $10.3 \%$ \\
Impedimento trabalhar/estudar/sair & $55.1 \%$ & $3.4 \%$ & $10.3 \%$ \\
Comportamentos sexuais forçados com recurso a ameaças, força física ou armas & $44.8 \%$ & $0 \%$ & $0 \%$ \\
Ameaçar/Chantagear através de TIC & $41.3 \%$ & $17.2 \%$ & $20.6 \%$ \\
Agredir física ou emocionalmente durante a gravidez & $34.4 \%$ & $0 \%$ & $0 \%$ \\
Estrangular/Asfixiar/Atropelar/Ferir com gravidade & $31 \%$ & $6.8 \%$ & $5 \%$ \\
Cortar/Queimar/Ferir & $27.6 \%$ & $3.4 \%$ & $0 \%$ \\
\hline & & &
\end{tabular}

\section{Evolução da sintomatologia das participantes}

O gráfico 1 apresenta as médias da sintomatologia geral obtidas através do OQ-45.2 nos três momentos de avaliação em cada grupo. Por razões que se prendem com a especificidade do grupo 1 , que se confrontou com a morte de um dos seus membros, iremos focar, num primeiro momento, a análise dos grupos $2,3 \mathrm{e}$ 4 e, num segundo momento, a análise específica do grupo 1.

No grupo 2 verificou-se uma diminuição do índice de sintomatologia geral do pré $(\mathrm{M}=60.0$; $\mathrm{DP}=16.97)$ para o pós-teste $(\mathrm{M}=47.0$; $\mathrm{DP}=22.63)$, tendo havido um agravamento, ainda que sem relevância clínica, deste para o follow up $(\mathrm{M}=54.0 ; \mathrm{DP}=22.63)$.

No grupo 3 houve uma diminuição do índice de sintomatologia geral, com relevância clínica, do pré $(\mathrm{M}=76.75 ; \mathrm{DP}=8.77)$ para o pós-teste $(\mathrm{M}=64.5$; $\mathrm{DP}=10.38)$ e uma ligeira subida, sem valoração clínica, deste para o follow up $(\mathrm{M}=66.0 ; \mathrm{DP}=8.76)$. 


\section{Gráfico 1}

Evolução da sintomatologia global (OQ-45.2)

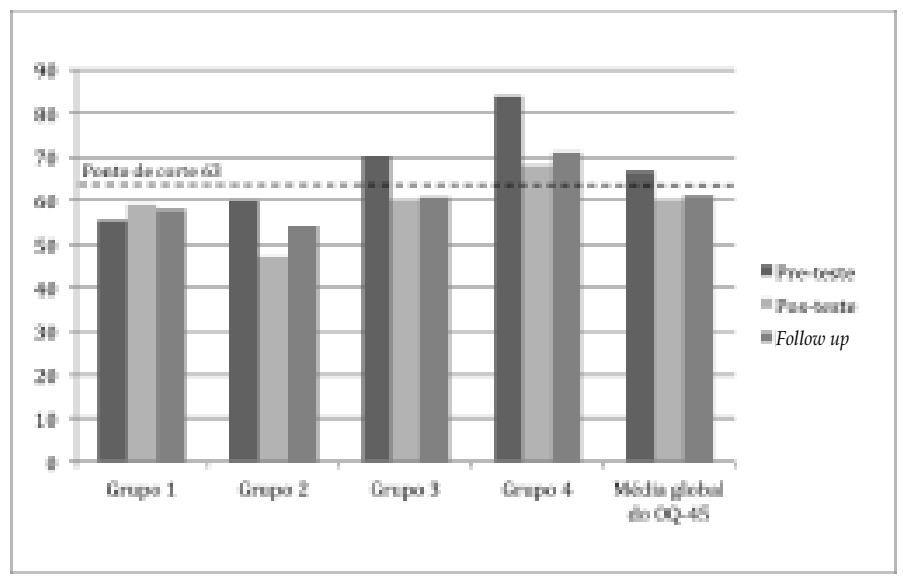

No grupo 4 verificou-se igualmente uma diminuição do índice de sintomatologia geral, com relevância clínica, do pré $(\mathrm{M}=84.0$; $\mathrm{DP}=22.11)$ para o pós-teste $(\mathrm{M}=68.0 ; \mathrm{DP}=18.74)$, tendo havido um aumento deste para o follow up $(\mathrm{M}=71.0$; $\mathrm{DP}=23.25)$.

No que concerne aos resultados do OQ-45.2 nestes 3 grupos, efetuou-se um teste de Friedman (Friedman's ANOVA) para detetar diferenças na evolução da sintomatologia destas participantes ao longo do tempo. Esta análise revelou diferenças estatisticamente significativas ao longo do tempo $\left(X^{2}(2)=8.727, p \leq 0.05\right)$, o que indicia um decréscimo da sintomatologia média entre o início $(\mathrm{M}=73.36$; $\mathrm{DP}=19.24$ ) e o fim da intervenção (no pós-teste: $\mathrm{M}=60.64 ; \mathrm{DP}=16.88$; no follow up $\mathrm{M}=63.27 ; \mathrm{DP}=18.29$ ).

No grupo 1 houve um aumento do índice de sintomatologia geral, ainda que sem relevância clínica, do pré $(\mathrm{M}=55.50 ; \mathrm{DP}=23.81)$ para o pós-teste $(\mathrm{M}=59.17 ; \mathrm{DP}=20.43)$ e um ligeiro decréscimo deste para o follow up $(\mathrm{M}=58.0$; $\mathrm{DP}=12.81$ ).

De um modo global, à entrada nos grupos terapêuticos, a média da sintomatologia geral encontrava-se num patamar clinicamente significativo, baixando para um patamar não-clínico no final do processo terapêutico. Note-se, no entanto, que o nível médio da sintomatologia geral aumentou ligeiramente do pós-teste para o follow up (cf. gráfico 1). 


\section{Evolução do empowerment das participantes}

O gráfico 2 apresenta a evolução das médias do empowerment obtidas através da EPPR nos três momentos de avaliação em cada grupo. Tal como na subsecção anterior, iremos focar esta análise, num primeiro momento, nos grupos 2 , 3 e 4 e, num segundo momento, no grupo 1 .

\section{Gráfico 2}

Evolução do empoderamento (EPPR)

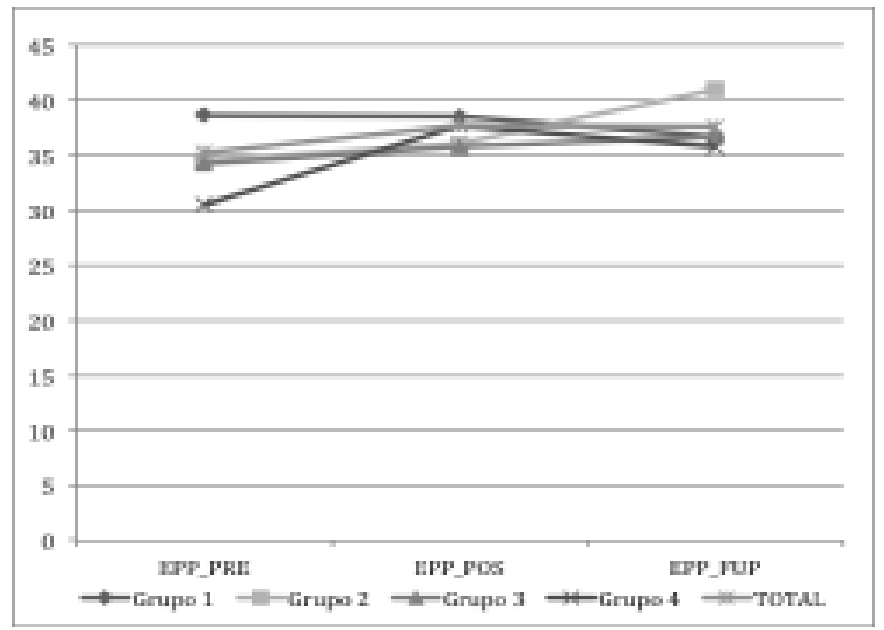

No grupo 2 verificou-se um aumento do empowerment do pré $(\mathrm{M}=34.5$; $\mathrm{DP}=2.12)$ para o pós-teste $(\mathrm{M}=38.5 ; \mathrm{DP}=6.22)$ e deste para o follow up $(\mathrm{M}=41.0$; $\mathrm{DP}=1.41$ ), acontecendo o mesmo no grupo 3 (no pré-teste: $\mathrm{M}=34.25 ; \mathrm{DP}=3.78$; no pós-teste: $\mathrm{M}=35.75$; $\mathrm{DP}=2.87$; no follow up $\mathrm{M}=36.75$; $\mathrm{DP}=7.85)$. No grupo 4 observou-se um aumento do empowerment do pré $(\mathrm{M}=30.5 ; \mathrm{DP}=6.86)$ para o pós-teste $(\mathrm{M}=37.75 ; \mathrm{DP}=3.30)$, mas uma diminuição deste para o follow up $(\mathrm{M}=35.75$; $\mathrm{DP}=9.61$ ).

No que concerne aos resultados do EPPR nestes 3 grupos, efetuou-se um teste de Friedman (Friedman's ANOVA) para detetar diferenças de médias do empowerment ao longo do tempo. Esta análise revelou diferenças estatisticamente significativas na evolução do empowerment ao longo do tempo $\left(X_{-}(2)=9.814, p \leq 0.05\right)$. Mais concretamente, a análise das médias nestes 3 grupos indica que houve uma evolução positiva, quando se compara o início $(\mathrm{M}=33.36 ; \mathrm{DP}=5.09)$ e o fim da intervenção (no pós-teste: $\mathrm{M}=37.27$; $\mathrm{DP}=3.66$; e no follow up: $\mathrm{M}=38.0 ; \mathrm{DP}=7.56$ ).

No grupo 1 observou-se um aumento do empowerment do pré (30.5) para o pós-teste (37.75), mas uma diminuição deste para o follow up (35.75). 


\section{Relação entre empowerment e mudança sintomatológica}

O estudo da relação entre a sintomatologia e o empowerment foi efetuado através da correlação de Spearman (focando-se a análise apenas nos grupos 2, 3 e 4). Observou-se uma relação significativa entre as variáveis. Em particular, quanto menor a sintomatologia no pós-teste, maior o empowerment no pós-teste $[r s=-.534, p$ (one-tailed) $\leq .05]$ e também no follow up [ $r s=-.681, p$ (one-tailed) $\leq .05]$. Para além disso, o empowerment evidenciado pelas participantes no início do grupo terapêutico, revelou-se inversamente correlacionado com a sintomatologia no follow up; isto é, quanto maior o empowerment à partida, menor a sintomatologia no follow up $[r s=-.589, p$ (one-tailed) $\leq .05]$.

\section{Discussão dos resultados}

Tendo em conta os resultados observados ao longo do processo terapêutico é possível afirmar que, no geral, foram feitos progressos muito relevantes ao nível do bem-estar psicológico e do empowerment das participantes e observados ganhos ao longo da intervenção terapêutica. Excetuando o grupo 1, que em consequência da morte de um dos seus membros se tornou compreensivelmente mais vulnerável, todos os outros, na generalidade, se fortaleceram, tendo contribuído para esta evidência, e segundo o relato das próprias, o facto de se ter gerado um clima de aceitação mútua e de partilha, promotor da coesão interindividual. Embora no grupo 4 não tenha sido possível obter as melhorias no índice de sintomatologia geral que se verificaram nos outros grupos (2 e 3), em virtude da gravidade da sintomatologia apresentada por algumas participantes (e.g., ideação suicida, risco de homicídio), considera-se que todas as participantes estão hoje mais conscientes das suas necessidades e, por consequência, das medidas que devem adotar para reduzir o risco clínico e de revitimação.

Todas as participantes, incluindo as do grupo 1, foram unânimes em considerar que a integração nos GRA constituiu uma mais-valia em termos da capacidade de refletir sobre as suas vivências pessoais e sociais e de se posicionar criticamente sobre o seu papel enquanto mulheres e enquanto vítimas. Na apreciação final dos ganhos, as participantes sublinharam o suporte mútuo como o aspeto mais empoderador da intervenção. O rompimento da ideia de vulnerabilidade única foi igualmente um fator reconhecido como muito positivo.

Com efeito, as participantes desenvolveram a sua capacidade de autoavaliação quer em relação a si próprias, quer em relação ao seu historial de vitimação, tomaram consciência dos seus papéis culturais e de género, aumentaram a sua perceção de autocontrolo e de eficácia pessoal, otimizaram as práticas de autocuidado, incrementaram as suas competências de comunicação e de gestão emocional e aumentaram os seus conhecimentos sobre os recursos disponíveis (pessoais e sociais) para lidar com a violência. Dentre estes recursos destacam-se aqueles 
que têm que ver com os seus direitos enquanto mulheres e enquanto cidadãs. $\mathrm{Na}$ realidade, o facto de os GRA as terem convidado a refletir sobre as suas pertenças sociais e sobre os seus lugares de poder, teve implicações ao nível da tomada de consciência sobre as suas possibilidades de ação e a sua capacidade de escolha. Este ganho foi mais evidente nas vítimas com menor escolaridade e mais idade, as mesmas que tendencialmente apresentam menor autonomização. À exceção das participantes do grupo 1, todas as outras concluíram o processo revelando mudanças subjetivas e objetivas. Note-se que do universo das oito participantes que ainda coabitavam com o agressor no início do processo terapêutico, quatro deixaram de o fazer. Avaliados os fatores que contribuíram para a sua decisão de abandonar a relação violenta, conclui-se que o sentimento percebido de injustiça e de desigualdade teve nestes casos em particular uma influência determinante.

Foi evidente, ao longo das sessões, a diminuição ou a cessação de alguns comportamentos de violência por parte dos companheiros ou ex-companheiros das participantes, tal como previsto na Literatura. Das 12 participantes que indicaram ser, aquando da inclusão nos grupos, vítimas de violência, seis deixaram de o ser. Em três casos observou-se uma diminuição significativa da violência. Esta alteração dos padrões de vitimação pode estar relacionada com a aquisição de competências pelas vítimas, ao nível da prevenção do risco e/ou da confrontação, bem como com o efeito indireto da participação das vítimas em programas de intervenção nos agressores (efeito dissuasor) (Cortés, 2011).

Não obstante a diminuição ou a cessação de alguns comportamentos de violência, há a sublinhar a tendência para a persistência de alguns outros, nomeadamente daqueles que configuram ações de controlo e perseguição, de risco acrescido. Denotou-se neste estudo uma diminuição das ações de violência física, mas uma persistência de atos que configuram situações de violência psicológica e emocional.

\section{Conclusões}

Tendo em conta os resultados descritos anteriormente, todos os grupos à exceção do 1 evoluíram, na generalidade, no sentido da diminuição da exposição à violência, da redução da sintomatologia clínica e do aumento do empowerment, concluindo-se assim pela eficácia dos GRA.

Para além destes ganhos, foi também notório o desenvolvimento da capacidade destas mulheres refletirem criticamente sobre os impactos da organização social de género nas suas trajetórias pessoais e nos seus percursos de vitimação, dado que se revelou, e segundo as próprias, determinante para as mudanças operadas.

Não obstante estas evidências que apontam para o sucesso genérico desta modalidade de intervenção psicoterapêutica, não pode deixar de analisar-se as questões que se revelaram menos eficazes neste projeto. Se é verdade que a promo- 
ção do empowerment favorece o bem-estar das vítimas de violência na intimidade, não é menos verdade que as pode tornar potencialmente mais vulneráveis às investidas dos agressores (Machado, 2004). Esta vulnerabilidade, que decorre muitas vezes da incapacidade dos agressores aceitarem e lidarem com a autonomização das vítimas, deve ser cuidadosamente avaliada em todas as fases do processo terapêutico. A monitorização constante do perigo deve por isso mesmo ser uma prioridade dos/as profissionais que conduzem a intervenção (Hilton, Harris e Rice, 2010), conhecidos que são os riscos da prática de comportamentos de stalking e de homicídio pelos agressores (companheiros e ex-companheiros), este último habitualmente premeditado e, por isso mesmo, difícil de evitar (Websdale, 2003).

Este esforço, contudo, não pode ser unilateral. Muitas vezes as ações de prevenção empreendidas pelas vítimas e pelos/as profissionais que as assistem não são acompanhadas de medidas judiciais céleres, inviabilizando-se parte da eficácia dos processos de intervenção. A este respeito, Sandra Walklate (2013) alerta para uma falha conceptual comum nas políticas públicas de combate à violência, particularmente no caso da violência contra as mulheres. Segundo a autora, a ênfase tem sido colocada no poder legislativo e não na promoção e implementação dos serviços de apoio às vítimas. Não obstante as mudanças legislativas implicarem uma mensagem sobre que comportamentos são inaceitáveis e responsabilizarem os/as ofensores/as, discute-se se o recurso à lei resolve efetivamente as necessidades das vítimas e se, por último, lhes restitui a dignidade.

Para que o direito constitua um recurso efetivo para a capacitação e autonomização das vítimas, é necessário que as políticas públicas promovam o encontro e a reciprocidade dos esforços empreendidos por profissionais de diferentes áreas no sentido da proteção e bem-estar das vítimas. São vários os estudos que apontam no sentido do discurso judiciário, por exemplo, ser propagador de uma visão estereotipada e preconceituosa das mulheres vítimas de violência na intimidade, havendo um fosso entre a consagração legal do princípio da Igualdade perante a Lei e a sua aplicação (Duarte, 2012). Na realidade, a Lei tem reproduzido muitos dos mitos associados às diferenças naturais entre os sexos, à pretensa harmonia do espaço familiar ou até à indissolubilidade do casamento (Dias, 2010), contribuindo tal visão para a desmobilização das vítimas no que à denúncia diz respeito e interferindo nas próprias decisões dos Tribunais. É conhecida, por exemplo, a desvalorização das alegações de violência no âmbito dos processos de regulação das responsabilidades parentais, o que reforça a descrença das vítimas no sistema judicial (Sottomayor, 2014).

O Estado deve reconhecer que as necessidades das vítimas de violência na intimidade se estendem para além do momento de crise e do plano individual, considerando o processo de vitimação como resultado de mecanismos sociais, culturais e políticos estruturalmente enraizados. Tal como descrito, a intervenção feminista revela-se eficaz por tomar em consideração o posicionamento social e as trajetórias de vida das vítimas. Adicionalmente, de acordo com os resultados do presente estudo, uma intervenção de cariz feminista traduz-se não só em ganho diretos, 
vivenciados ao nível do empowerment das participantes e cessação dos comportamentos de violência sofridos, como também em ganhos indiretos, experienciados ao nível da diminuição da sintomatologia e aumento do bem-estar psicológico.

Os resultados deste estudo demonstram como o empowerment e a capacitação para a reflexão deverão ser entendidos como objetivos fundamentais de atuação e intervenção com as vítimas. Uma vez que as estratégias governamentais têm o poder de legitimar ou excluir o sofrimento das vítimas, ao considerá-lo (ou não) abrangível pelas medidas das políticas nacionais (Walklate, 2013), julga-se essencial a inclusão deste tipo de intervenção nos planos de combate à violência de género. É neste sentido que se entende a dignidade da vítima e a sua capacidade de tomada de decisão como eixos basilares de uma política orientada para os direitos.

\section{Referências}

Allen, Karen Neuman; Wozniak, Danielle F. (2013), «The Integration of Healing Rituals in Group Treatment for Women Survivors of Domestic Violence», Social Work in Mental Health, volume 12, número 1, pp. 52-68.

Almeida, Leandro; Freire, Teresa (1997), Metodologia de Investigação em Psicologia e Educação, Braga, APPORT.

Amâncio, Lígia (1994), Masculino e Feminino. A Construção Social da Diferença, Porto, Afrontamento.

Bourdieu, Pierre (1999), A Dominação Masculina, Oeiras, Celta.

Comissão para a Cidadania e Igualdade de Género (2011), Violência Doméstica: Intervenção em grupo com mulheres vitimas. Manual para Profissionais, Porto, Comissão para a Cidadania e Igualdade de Género.

Conselho da Europa (2013), Convenção do Conselho da Europa para a Prevenção e Combate à Violência contra as Mulheres e a Violência Doméstica, Lisboa, Comissão para a Cidadania e Igualdade de Género.

Cortés, Neus; Serra, Júlia (Eds.) (2011), Intervención grupal en violencia sexista. Experiencia, investigación y evaluación, Barcelona, Herder.

Davies, Pamela; Francis, Peter; Jupp, Victor (2003), Victimology, victimisation and public policy, Hampshire, Palgrave.

Dias, Isabel (2010), «Violência doméstica e justiça», Sociologia: Revista do Departamento de Sociologia da FLUP, volume XX, pp. 245-262.

Dias, Isabel; Guerreiro, Maria das Dores (2010), «Violência na família. (In)visibilidades de um velho problema social», in António Dornelas, Luísa Oliveira, Luísa Veloso, Maria das Dores Guerreiro (org.), Portugal Invisivel, Lisboa, Editora Mundos Sociais, pp. 155-172.

Duarte, Madalena (2012), «O lugar do Direito nas políticas contra a violência doméstica», Ex aequo, n.. 25, pp. 59-73, [em linha] disponível em endereço http://www. scielo.oces.mctes.pt / scielo.php? pid=S0874-55602012000100006\&script $=$ sci arttext\&tlng=pt [consultado em 10 de dezembro de 2014].

Enns, Carolyn Zerbe (2004), Feminist theories and feminist psychotherapies. Origins, themes and diversity, Oxford, The Haworth Press.

Ferreira, Célia; Matos, Marlene (2013), «Violência doméstica e stalking pós-rutura: dinâmicas, coping e impacto psicossocial na vítima» Psicologia, volume 27, número 2, pp. 81-106. 
Ferreira, Virgínia (1998), «Engendering Portugal: Social Change, State Politics and Women's Social Mobilization», in António Costa Pinto (Ed.), Contemporary Portugal: Politics, Society and Culture, Stanford, Stanford University Press, pp. 162-188.

European Union Agency for Fundamental Rights (2014), Violence against Women: an EUwide survey, Luxembourg, Publications Office of the European Union.

Hilton, Zoe; Harris, Grant; Rice, Marnie (2010), Risk Assessment for Domestically Violent Men: Tools for Criminal Justice, Offender Intervention, and Victim Services, Washington, American Psychological Association.

IBM Corporation (2013), IBM SPSS Statistics for Windows, Version 22.0, Armonk, IBM Corporation.

Jacobson, Neil; Truax, Paula (1991), «Clinical significance: A statistical approach to defining meaningful change in psychotherapy research», Journal of Consulting and Clinical Psychology, volume 59, número 1, pp. 12-19.

Johnson, Dawn M.; Worell, Judith; Chandler, Redonna K. (2005), «Assessing psychological health and empowerment in women: The Personal Progress Scale Revised», Women E Health, volume 41, número 1, pp. 109-129.

Jones, Patricia S.; Meleis, Afaf I. (1993), «Health is empowerment», Advanced Nursing Science, volume 15, número 3, pp. 1-14.

Lambert, Michael J.; Burlingame, Gary M.; Umphress, Val; Hansen, Nathan B.; Vermeersch, David A.; Clouse, Glenn C.; Yanchar, Stephen (1996), «The reliability and validity of the Outcome Questionnaire», Clinical Psychology and Psychotherapy, volume 3, número 4, pp. 249-258.

Lisboa, Manuel; Carmo, Isabel; Vicente, Luísa; Nóvoa, António; Barros, Pedro P.; Silva, Sofia; Roque, Ana; Amândio, Sofia (2006), Prevenir ou Remediar - Os Custos Sociais e Económicos da Violência Contra as Mulheres, Lisboa, Colibri.

Loureiro, Cecília; Pinheiro, Fábia; Neves, Sofia (2012), «Intervenção psicológica em grupo com mulheres vítimas de violência de género na intimidade», in Sofia Neves, (coord.) Intervenção psicológica e social com vítimas, Coimbra, Almedina, pp. 39-62.

Machado, Carla (2004), «Intervenção psicológica com vítimas de crimes: Dilemas teóricos, técnicos e emocionais», International Journal of Clinical and Health Psychology, volume 4, número 2, pp. 399-411.

Machado, Paulo; Fassnacht, Daniel (2014), «The Portuguese version of the Outcome Questionnaire (OQ-45): Normative data, reliability and clinical significance cut-offs scores», Psychology and Psychotherapy: Theory, Research and Practice. DOI:10.1111/ papt.12048.

Matos, Marlene; Machado, Andreia; Santos, Anita; Machado, Carla (2012), «Intervenção em grupo com vítimas de violência doméstica: Uma revisão da sua eficácia», Análise Psicológica, volume 30, n.. 1-2, [em linha] disponível em endereço http:/ / www.scielo. gpeari.mctes.pt/scielo.php?pid=S0870-82312012000100008\&script=sci_arttext [consultado em 10 de setembro de 2014].

Ministério da Administração Interna (2014), Relatório Anual de Segurança Interna 2013, [em linha] disponível em endereço http://www.portugal.gov.pt/media/1391220/ RASI\%202013.pdf [consultado em 1 de dezembro de 2014].

Neves, Sofia; Cunha, Carla; Grangeia, Helena; Correia, Ariana (2014a), Inventário sobre Vivências Violentas nas Relações Íntimas (Versão para investigação não publicada).

Neves, Sofia; Cunha, Carla; Grangeia, Helena; Correia, Ariana (2014b). Escala de Progresso Pessoal Revista (Versão para investigação não publicada).

Neves, Sofia; Nogueira, Conceição (2003), «A Psicologia Feminista e a Violência contra as Mulheres na Intimidade: A (Re)Construção dos Espaços Terapêuticos», Psicologia e Sociedade, volume 15, número 2, pp. 43-64. 
Neves, Sofia; Nogueira, Conceição (2004), «Terapias Feministas, Intervenção Psicológica e Violências na Intimidade: Uma leitura feminista crítica», Psychologica, volume 36, pp. 15-32.

Neves, Sofia; Nogueira, Conceição (2005), «Metodologias Feministas: Reflexividade ao serviço da Investigação nas Ciências Sociais», Psicologia: Reflexão e Crítica, volume 18, número 3, pp. 408-412.

Observatório das Mulheres Assassinadas (2014), Comunicado sobre o Relatório intercalar do OMA (jan. a jun 2014), [em linha] disponível em endereço http://www.umarfeminis mos.org/index.php/inicio/15/842 [consultado em 1 de dezembro de 2014].

Rappaport, Julian (1984), «Studies in empowerment: introduction to issues», Prevention in Human Services, volume 3, número 2-3, pp. 1-7.

Sottomayor, Clara (2014), Temas de Direito das Crianças, Coimbra: Almedina.

Walker, Lenore (1999), «Psychology and Domestic Violence around the World», American Psychologist, volume 54, número 1, pp. 21-29.

Walker, Lenore (2000), Abused women and survivor therapy. A practical guide for the Therapist, Washington, American Psychological Association.

Walklate, Sandra (2013), «Victims and european policy initiatives», in Stephanie Petrie (ed.), Controversies in policy research: Critical analysis for a new era of austerity and privation, Hampshire, Palgrave, pp. 16-35.

Websdale, Neil (2003), «Reviewing domestic violence deaths», National Institute of Justice Journal, número 250, pp. 26-31.

Worell, Judith; Chandler, Redonna K. (1998), The Personal Progress Scale-Revised (PPS-R). Unpublished manuscript.

Worell, Judith; Remer, Pamela (2003), Feminist perspectives in therapy. Empowering diverse women, New Jersey, Wiley.

Sofia Neves é Licenciada em Psicologia e Doutorada em Psicologia Social pela Universidade do Minho (UM). É atualmente docente e investigadora do Instituto Universitário da Maia (ISMAI). asneves@docentes.ismai.pt

Carla Cunha é Licenciada em Psicologia pelo ISMAI e Mestre e Doutora em Psicologia Clínica pela UM. É atualmente docente e investigadora do ISMAI. ccunha@docentes.ismai.pt

Helena Grangeia é Licenciada e Doutorada em Psicologia da Justiça pela UM. É atualmente docente e investigadora do ISMAI. hgrangeia@docentes.ismai.pt

Ariana Correia é Licenciada em Psicologia e Mestre em Psicologia Clínica e da Saúde pelo ISMAI. ariana.pinto.correia@gmail.com

University Institute of Maia (ISMAI)

Department of Social and Behavioral Sciences

Avenida Carlos Oliveira - Castêlo da Maia

4475-690 Avioso S. Pedro

Portugal

Artigo recebido em 29 de setembro de 2014 e aceite para publicação em 27 de dezembro de 2014. 